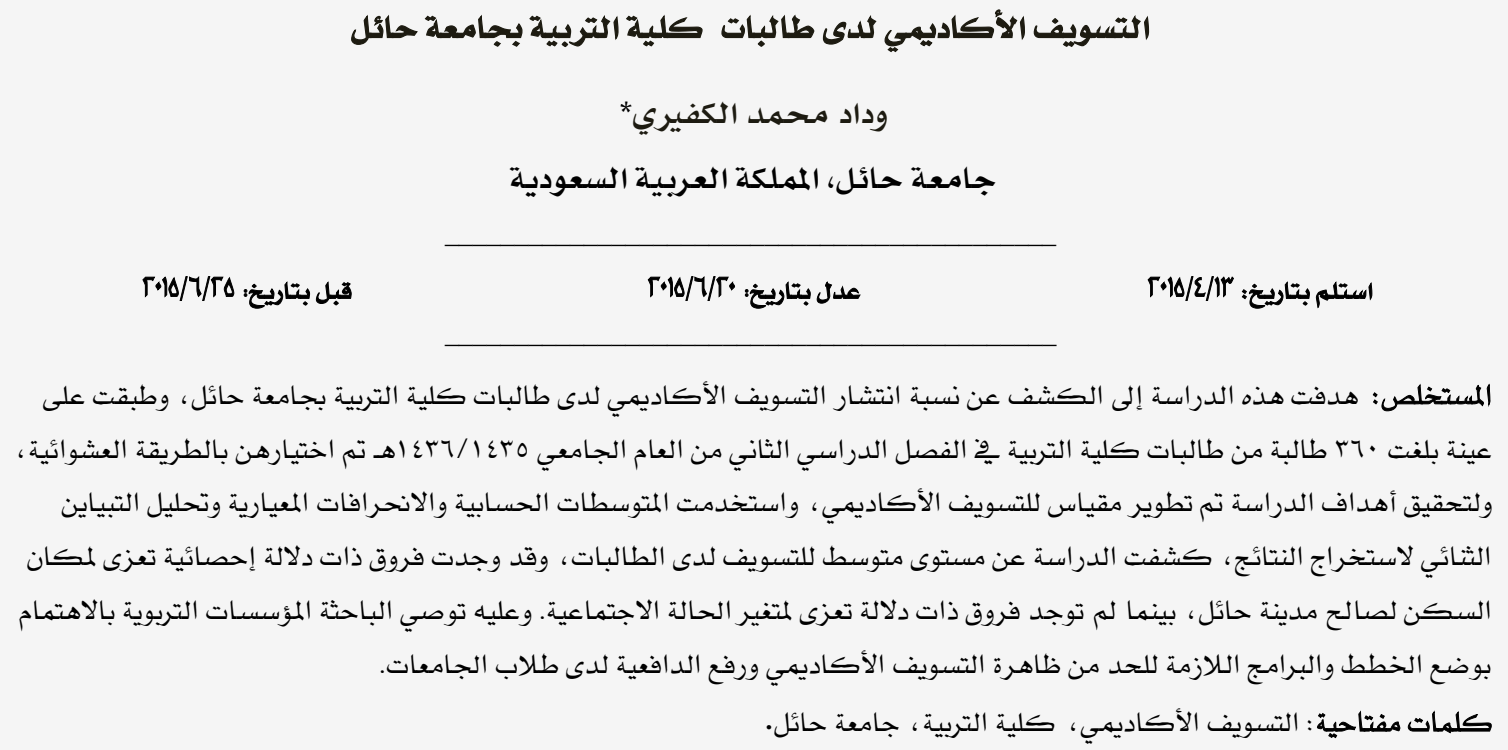

\title{
Academic Procrastination among Students of the Faculty of Education at Hail University
}

Wedad M. Alkferi*

Hail University, Kingdom of Saudi Arabia

\begin{abstract}
This study aimed to investigate the prevalence of academic procrastination among students in the Faculty of Education at the University of Hail, and applied on a sample of 360 female students in the second semester of the academic year 2014/2015 selected randomly. To achieve the objectives of the study a measure of academic procrastination was developed. The researcher used mean \& standard deviation, to conclude the findings. The findings of the study showed that an average level of procrastination among female students. Differences were found statistically significant due to the place of residence for the city of Hail, while there were no significant due to the variable marital status differences. The researcher recommended worthwhile educational institutions develop plans and programs needed to reduce the phenomenon of academic procrastination and raise the motivation of university students.
\end{abstract}

Keywords: Academic procrastination, Faculty of Education, University of Hail.

*fathy@squ.edu.om 
الأكاديمي بأنه التأخير المتعمد أو التأجيل

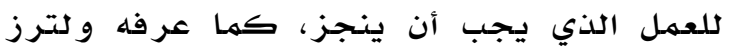

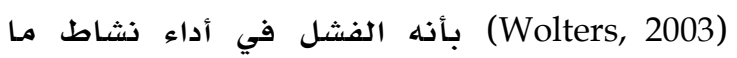
وفق الزمن الهرغوب وتأجيله حتى آخر دقيقة،

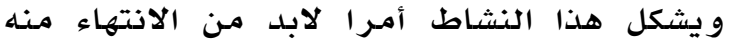
و صو لا الى الارتياح. و التسويف الأكاديمي تأخير القيام بالهمام الأكاديمية نتيجة التناقض بورلم بين

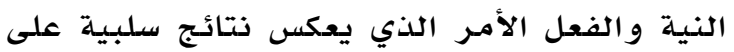

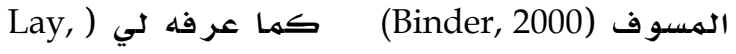
1986) بأنه الهيل الكلاعقلاني لتأخير الهمهمات التي

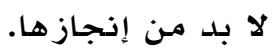

و يشار إلى أن التسويف ظاهرة لهم تلق إجماعا مـن

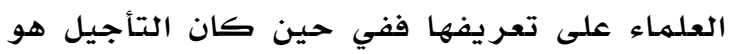
الهكون الحاسهم عند البعض (Piccarelli, 2003) فإن البعض الآخر عد القلق المؤدي إلى هذا التأجيل (Rothblum et al., هو المكون الأساسي للتسويف التهوديف

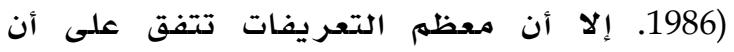

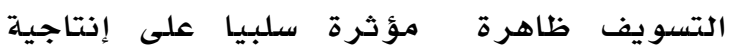
الفرد و مستوى ثقته في نفسه، و صدحته النفسية. وتختلف وجهات النظر في تفسير ظاهرة

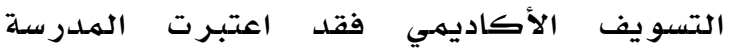

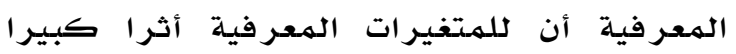

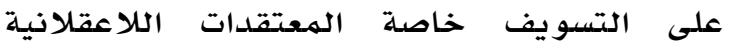
،(Beswick, Rothblum \& Mann, 1988)

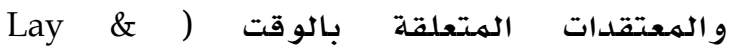
(Schouwenberg, 1993 وتقدير الذات (Beswick) et al., 1988) التسويف لاهرة متعلمـة تنشأ نتيجة تفضيل الإنسان النشاطات السارة، والمكافآت الفورية، في

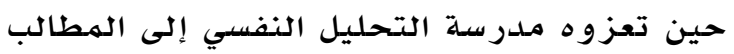

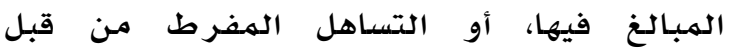
الوالدين. (McCown, Petzel \& Rupert, 1987) و للتسويف أنواع كثيرة من أهمها التسويف في

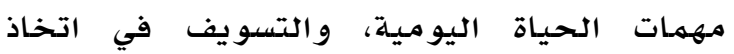
Milgram, القرارات، والتسويف الأكاديمي. (Batori \& Mowrer, 1983)

و قد بين العنزي و الدغيه (r..r) أن هذه الظاهرة منتشرة بين الهتعلمين، و وتتمثثل في تأخير

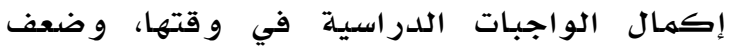

لا شك بأن تأجيل المهمات والواجبات لأمـر

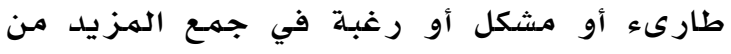
المعلومات، أو نتيجلة ترتيب الأولويات و إعطاء الأولوية لأداء بعض الهمهمات دون غيرها ها مـها

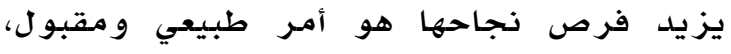
و عده فيراري في الحالة الأخيرة شكلا من في أشكال الإتقان (Ferrari,1991). إلا أن أن التأجيل المسيتهـر

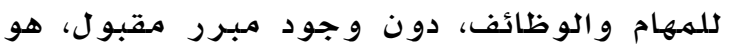
أمـر مشكل ويعوق أداء الههام، ويقلل من فرصو نجاحها. و لما كان التحصيل الأكاديمي هو الأداة التي من خلالها تتمكن الجـامعة أوالهؤسسة التعليمية التهية

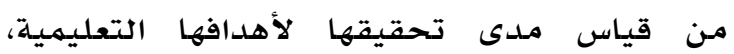

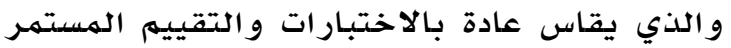

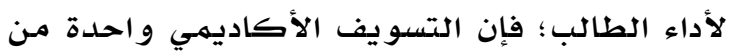
أخطر الهشاكل في الحياة اليومية، والبيئات

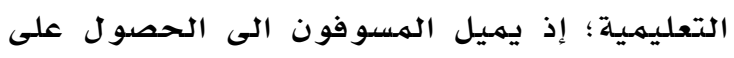

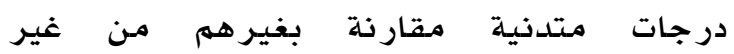

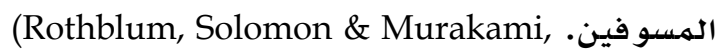
1986, Tice \& Baumeister, 1997)

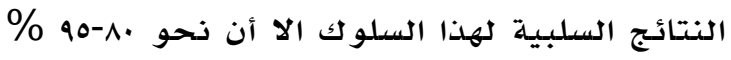

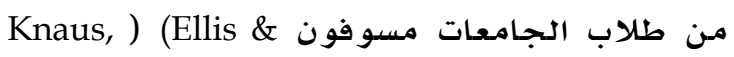

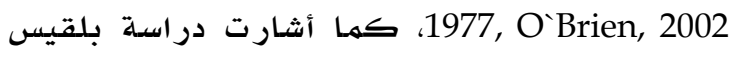

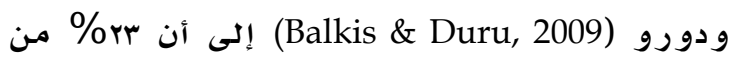

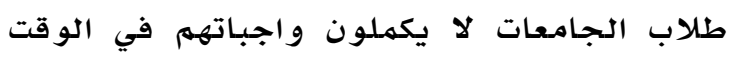
الهحدد و يؤجلونها إلى وقت لاحق، وفي دراسلة

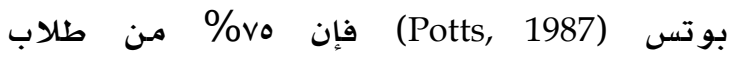
الجامعات يسوفون، كما أن دراسـة أوزر و فيراري

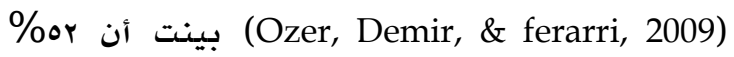
من الطلاب يؤجلون. و يشير جاكسون وزمـلاؤه (Jackson etal) المشار

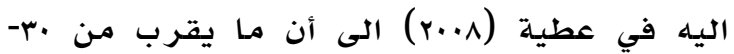

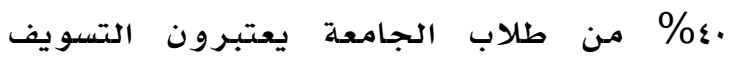

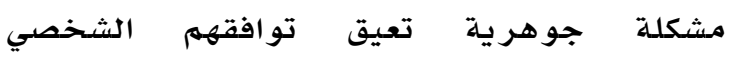
و الوظيفي. وبحسب ونج (Wong) فإن إن التلكؤ تأجيل لشيء ما إلى وقت لاحق وهو بوني وهيق الفرد

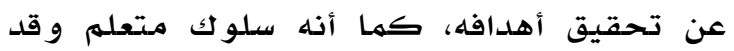
يكون شكلا من أشكال الهقاومة لكلأشياء التي تعد

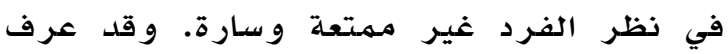

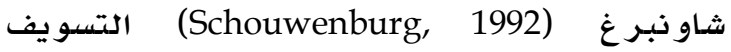


وبالتالي الشعور بالإحباط والذنب. ويعتبر

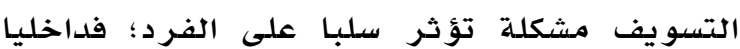
يشعر بالندم واليأس ولوم الذات، أما خارجيا

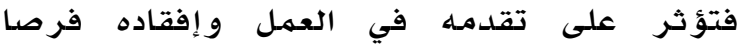
كثيرة في الحياة (عطية، ^..r). و وحسب العنزي

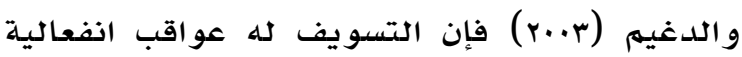

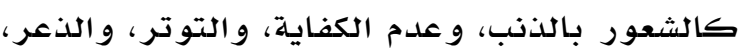
وصعوبات في النوم، إضافة الى القلق، ومـمارسة سلوكيات التدخين و الكحول.

وقد بحثت العديد من الدراسات الأجنبية ظاهرة التسويف؛ في دراسة أجريت من قبل تشعانغ (Chang, 2014) بعنوان الكمالية، القلق، التسويف الأكاديمي ودور

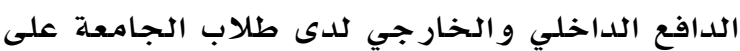
عينة مكونة من هبr طالبا من طلاب جامعة و لاية

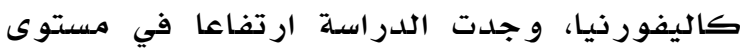
التسويف الأكاديمي والقلق لدى الطلاب وارتباط

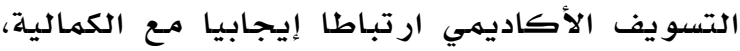

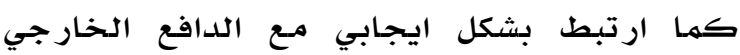
و بشكل سلبي مـع الدافع الداخلي.

ودراسـة بعنوان علاقة الهعتقدات العقلانية حول

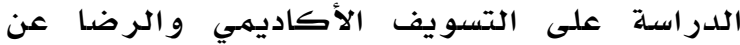

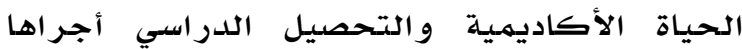
دورو و بلقيس (Duru \& Balkis, 2013) على عينة

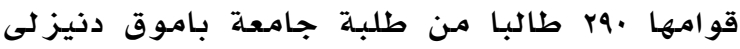
التركية، كشفت الدراسلة عن وجود علاقة سلبية

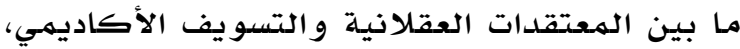

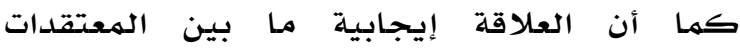
العقلانية والر ضـا عن الحياة الأكاديمية والإنجاز الأكاديمي.

وفي دراسة أجريت من قبل كندي وتوكمان (Kennedy \& Tuckman, 2010) فرضية تأثير الهماطلة الأكاديمية على الهعدل التراكمي لدى طلاب السنـة الأولى بجامعة و لاية

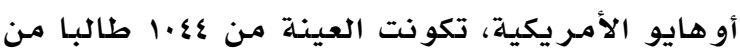

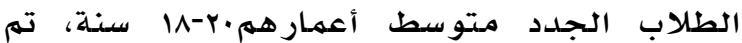
اختيار هم بالطريقة العشوائية و استخدم مقياس توكمان للتسويف الأكاديمي لقياس التسويف الأكاديمي (توكمان، 1991) و المكون منوان 17 فقرة.
الإستعداد للاختبارات، والدرراسلة لأوقات غير كافية . الإ.

و تتنوع أسباب التسويف فقد بينت الدراسات أن

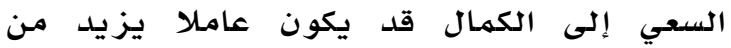
احتمالية التسويف ؛إذ يضع السـاعون إلى الكمال

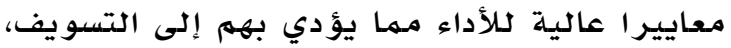
كما قد يكون سبب التسويف فكرة أنني أعمل بشكل أفضل تحت الضغط محاولة من المسوف

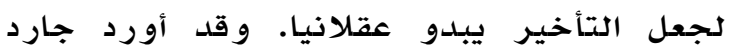
(Gard,1999)

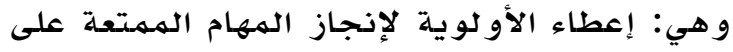

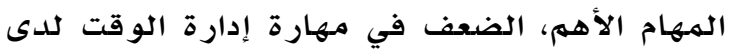
الهسوف، السعي نحو الكمالية، التهرب من الههام

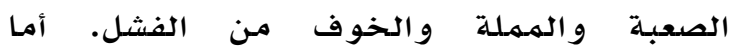

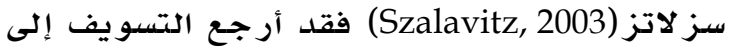

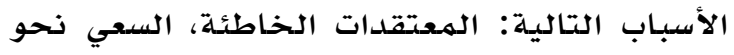

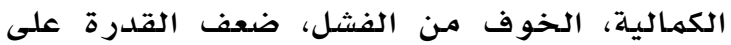

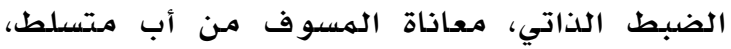
قلق انجاز المهمهة، والاكتئياب.

و فيما يتعلق بأسباب التسويف للى طالبات جامعة

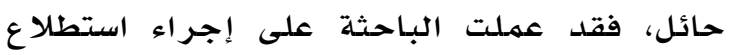
للطالبات حول أسباب التسويف الأكاديهي لديهن، حيث كشف الاستطلاع أن السبب الأول للتسويف

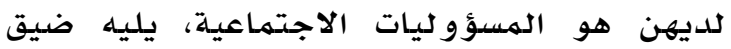

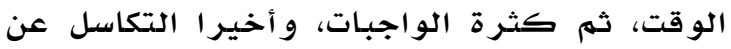

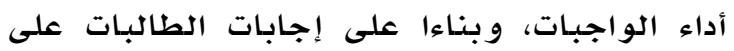
الاستطلاع توقعت الباحثة أن تكون الحالة

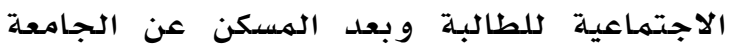

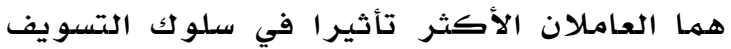
الأكاديمي لدى الطالبات.

و للتسويف آثاره السلبية على الحياة الأكاديمية

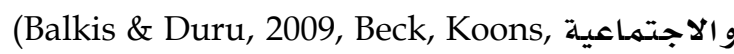
(\& Migram, 2000) المنخفض (Tice \& Baumeister, ،1997) ، و الانقطاع الهُ عن حضور الحصص الدراسية، ( Semb, Glick \&) و وتظهر الدر اسـات أن تأجيل

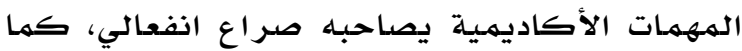

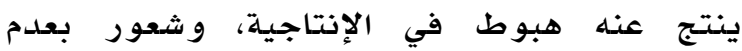
الكفاية، وسوء الإدارة، وعدم الإلتزام بالمواعيد ولإديد 
التسويف أعلى للىى طلبـة السنة الر ابعة منـه لدى

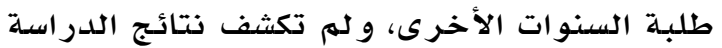
عن فروق دالة إحصائيا تعزى لمتغيري الجنس و التخصص الأكاديهي.

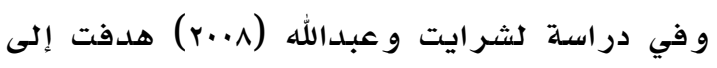
التعرف على علاقة التلكؤ الأكاديمي بالدافعية للإِنجاز و الفاعليـة الذاتية. تكونت عينـة الدراسلة

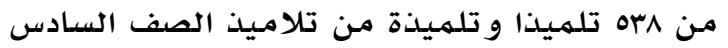
في محافظة الإسكندرية. كشفت نتائج الدراسـة عن فروق دالة إحصائيا بين متوسط درجات

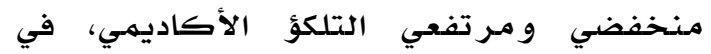
الدافعية لإنجاز والفاعلية الذاتية لصالح منخفضي التلكؤ الأكاديمي.

أما دراسـة جر ادات (Jaradat, 2004) فقد هدفت إلى الكشف عن علاقة قلق الاختبار بالتسويف

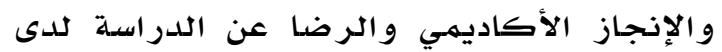

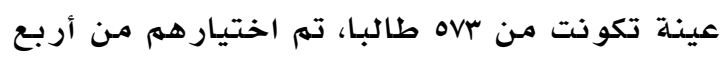

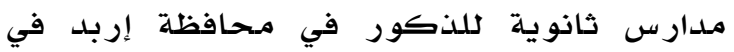
الأردن. كشفت نتائج الدراسة عن علاقة إيجابية دالة إحصدئيا بين قلق الاختبار و والتسويف الأكاديمي، و علاقة سلبية دالة إحصائيا بين قلق

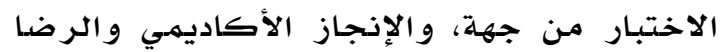

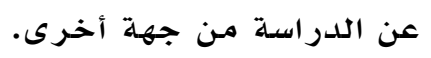

كما أجرى هلال و الحسيني (ع...r)، دراسة هدفت إلى الكشف عن التلكؤ الأكاديهي و علاقته ببعض المتغيرات النفسية. تكونت عينة الدراسـة الدية

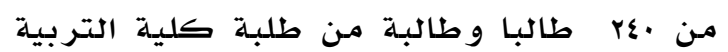

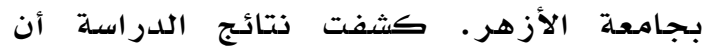
الطلاب أكثر ميلا للتلكؤ الأكاديمي من هن الطالبات، و أن الطلبة منخفضي التلكؤ أكثر رضا

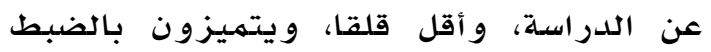

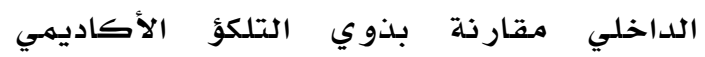

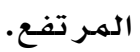

وأخيرا أجرى العنزي و الدغيهم (r...r) دراسـة هدفت إلى الكشف عن سلو كك التسويف الأكاديمي و علاقته ببعض متغير ات الشخصية، على عينة من إنى

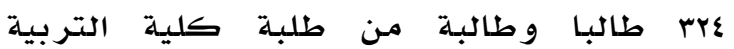
الأسساسية بالكويت. وقد كثفت نتائج الدراسـة عن عدم وجود فروق دالة إحصائيا بين الذكور
تبين من الدراسـة العلاقة العكسية السلبية بين التسويف الأكاديمي و المعدل التر اكمي. أمـا عربيا فقد أجريت دراسـات حول التسويف

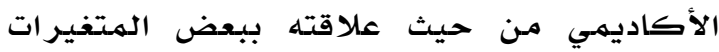

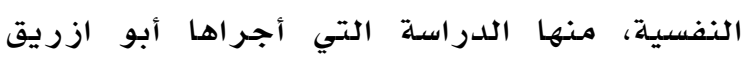

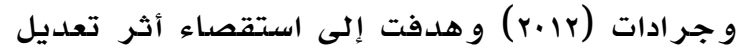

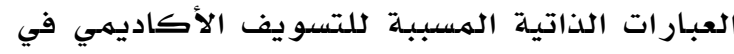
تخفيض التسويف الأكاديمي و تحسين الفاعلية الذاتية الأكاديمية لدى عينه من طلاب الصف وله العاشر،استخلدم فيها مقياسا للتسويف الأكاديهي،

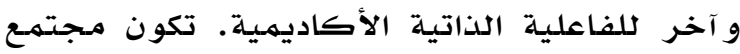

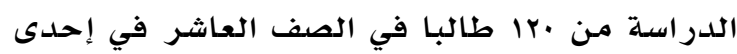

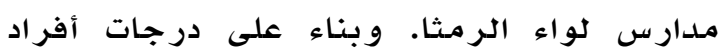
مجتمـع الدراسـة على مقياس التسويف الأكاديهي

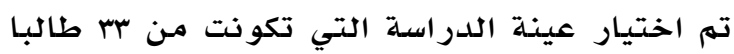

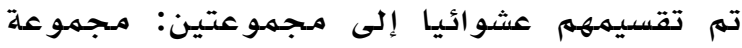

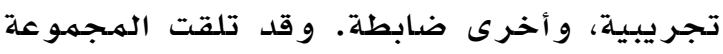

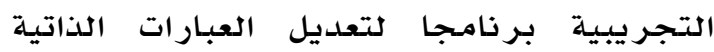
المنتتجة للتسويف الأكاديمي، والذي تكون مـن

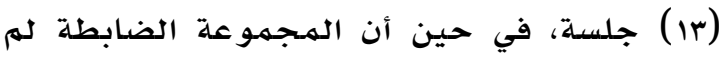

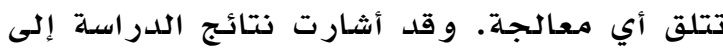

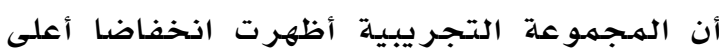

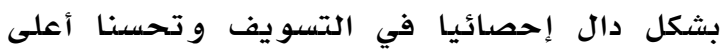
بشكل دال في الفاعلية الذاتية في كل من الفيات

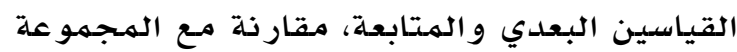

الضابطة.

دراسـة أخرى لأبو غزال (r.r) هدفت إلى التعرف على مدى انتشار التسويف الأكاديهي و أسبابه من وجهة نظر الطلبة الجامعيين، و فيها

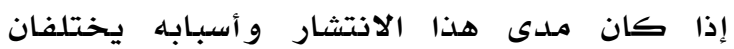
باختلاف جنس الطالب و مستواه الدراه

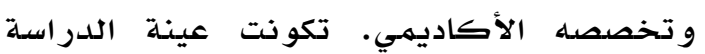

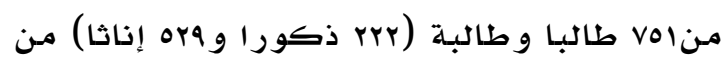
جميع كليات جامعة الير موك. كشفت نتائج

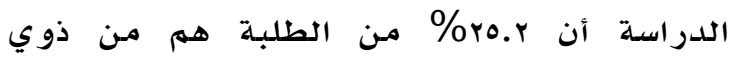

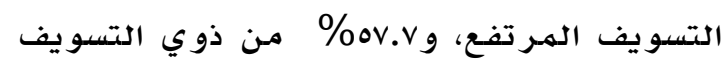

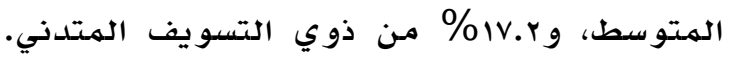

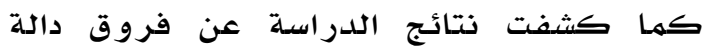
إحصائيا في انتشار التسويف الأكاديمي تعزى العى

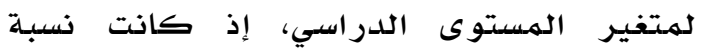


التسويف الأكاديمي لدى طالبات الكلية- وقوفا

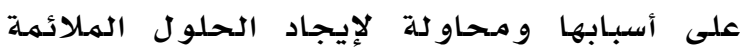
للحد من انتشار هذه الظاهرة؛ فالطالب السليهم

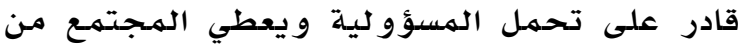

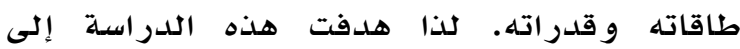

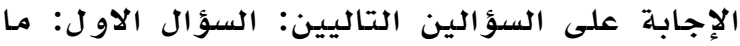
نسبة انتشار التسويف الأكاديهي لدئ لدو طالبات جامعة حائلى

السؤال الثاني: هل تختلف نسبـة انتشار التسدويف الاكاديمي باختلاف مكان السكن والحالة الاجتماعية؟ الادي

أهمية الدراسة

تبرز أهمية هذه الدراسلة كونها تبحث ظاهرة خطيرة لقيت اهتماما لدى كثير من اهن الباحثين التربوين في العاله، وكون هذه الظاهرة تهدد

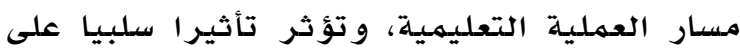
صححة الفرد، إذ تبين من الدراسات أن للتسدويف

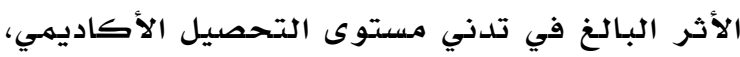
وانتشار القلق وفقدان الثقة بالنفس و مشاعر الذنب لدى المسوفين. كما تبرز أهميتها كونها

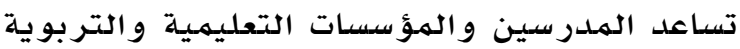
و المجتمهـع بشكل عام، في الإطلاع على مدى انتشار

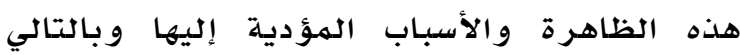

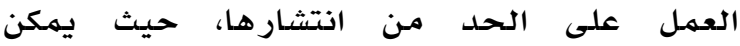
الإستفادة من نتائج هذه الدر اسـة في مـالهـال الإرشـاد

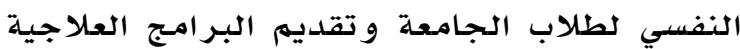
اللازمة لتنمية دافعيتهم نحو الإنجاز، كما وتبرز

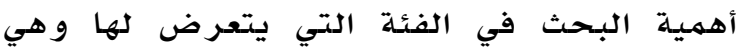
طلاب الجامعات والذين يشكلون عمادا لهستقبل

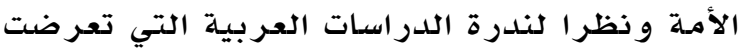

لههذا الموضوض.

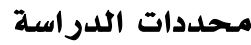

اقتصرت الدراسلة على طالبات جامعة حائل الفرع الرئيسي المسجلات للفصل الدراسي الثاني لعام

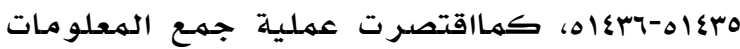
على الأداة المستخدمة في الدراسلة والاستجابة على فقر اتها من قبل أفر اد العينـة.
و الإناث على مقياس التسويف الأكاديهي، كها كشفت عن علاقة سلبية دالة إحصائيا بين التسويف الدراسي من جهة، والثقة بالنفس

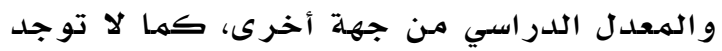

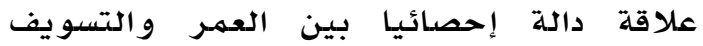

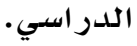

يلاحظ مـ خلال استعراض الأدب التربوي و نتائج الدراسات السابقة التي تم عرضها، ارتفاع

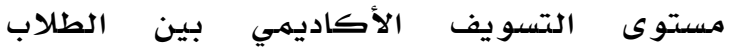
و ارتباطه ارتباطا عكسيا بالمعدل التراكهي

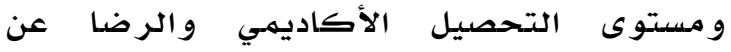

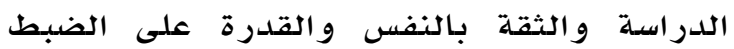
الداخلي وارتباطه ارتباطا ايجابيا بالقلق. ولهم

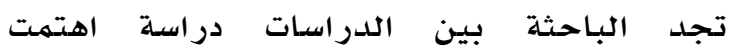
بمتغير ات مكان السكن أو الحالة الاجتهماعية، مـا لها

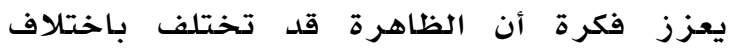

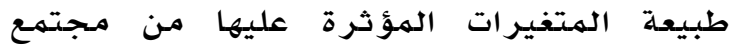

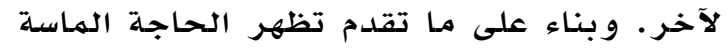

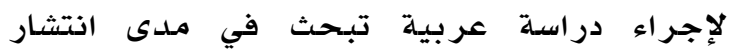
التسويف الأكاديمي في ضوء متتغيري مكان

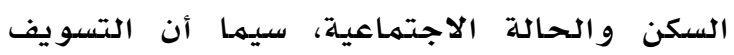

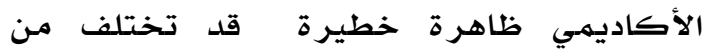
مجتمع لآخر من حيث الانتشار و المستوى.

مشكلة الدراسة و أسئلتها

تبين للباحثة من خلال عملها همضو هيئة تدريس في جامعـة حائل مؤشرات تدل على انتشار ظاهرة التسويف الأكاديمي لدى طالبات الجامعة ؛

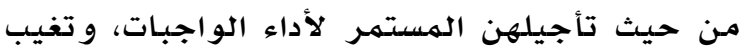

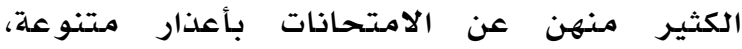

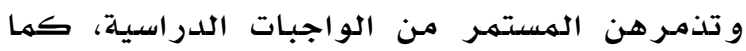
لاحظت سفر بعض الطالبات إلى الجامعة يوميا من مسافات بعيدة تصل أحيانا إلى . .باكم

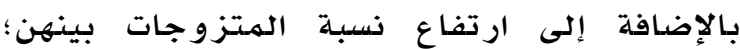
و لكون التسويف ظاهرة لها آثارها السلبية على لهـ

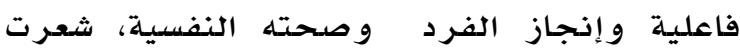
الباحثة بالمسؤو لية بضرورة إجراء دراسة تبحث في انتشار الظاهرة في ضوء متيفيري مكان بان السكن و الحالة الإجتماعية- حيث توقعت الباحثة أن يكون

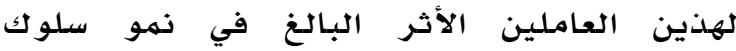




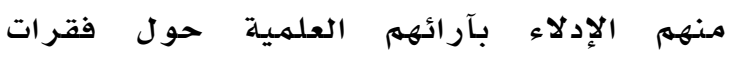

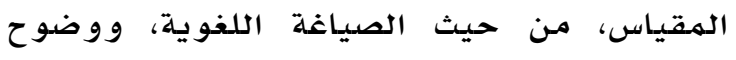

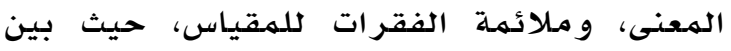

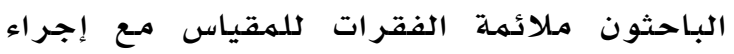
بعض التعديلات الطفيفة على الصياغة اللغوية.

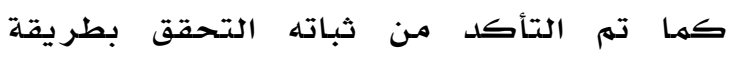

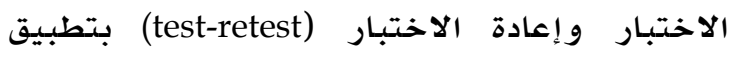

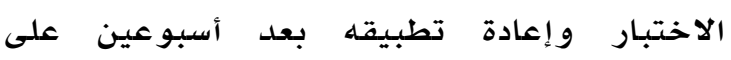

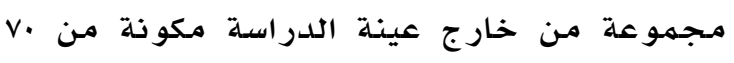

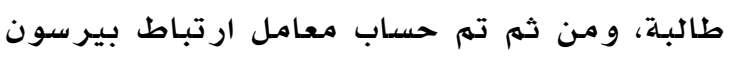

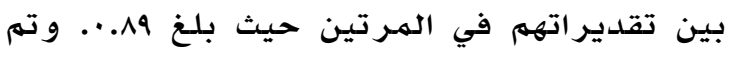

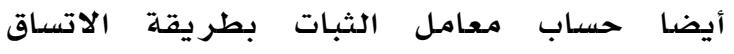

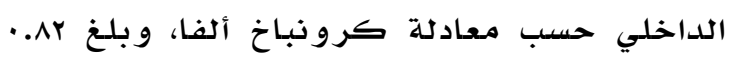

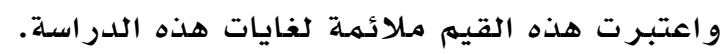

\section{تصحيح المقياس}

يتكون الهقياس من الم فقرة تتم الإجابة عليها

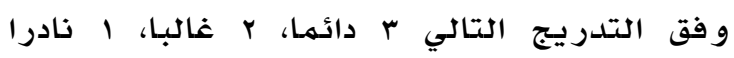
وصفر مطلقا، وقد تم حساب المستويات لفقرات

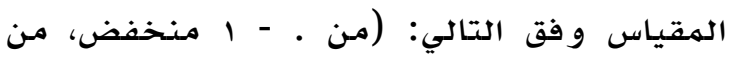

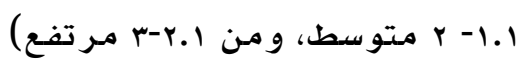

إجراءات الدراسة بعد الانتهاء من تطوير أداة الدراسة والتأكد من

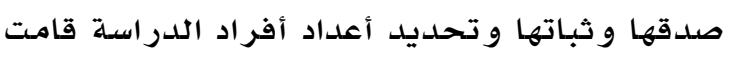
الباحثة بتوزيع المقياس على الطالبات في قاعة ولهابل

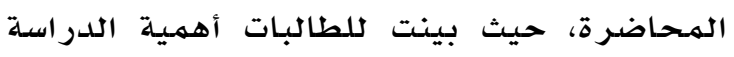

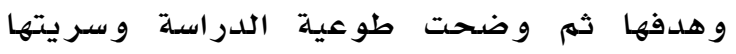

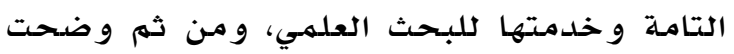

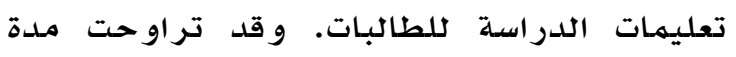

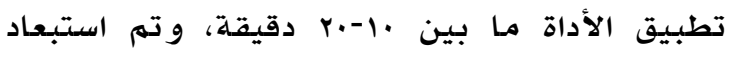
الاستبانات التي لهم تحقق الشروط بسبب لهب نقص

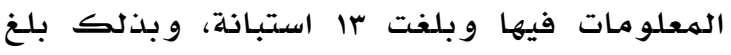

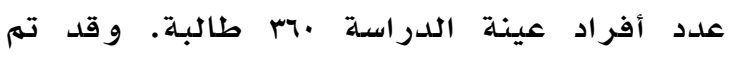
تصحيح الاستجابات وفق تدريج رباعي من ل.-r

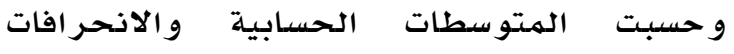
المعيارية، كما استخدم تحليل التباين الثنائي للإجابـة على سؤالي الدر اسلة.

\section{التعريفات الإجر ائية}

التسويف الأكاديمي: تأجيل الطالب دون مبرر إنهاء الواجبات الدراسية المطلوبة منه حتى آخر

لحظة.

طالبات كلية التربية بجامعة حائل: وهن جميع الطالبات المسجلات للفصل الدراسي لئل الثاني

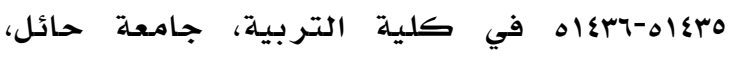
الفر ع الر ئيسي.

\section{الطريقة والإجر اءات}

\section{مجتمـع الدراسة}

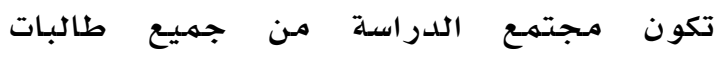

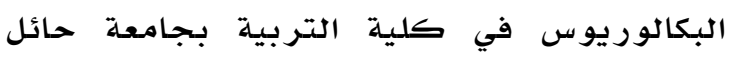

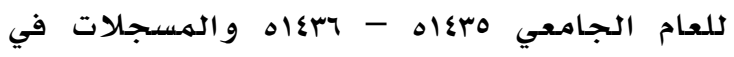

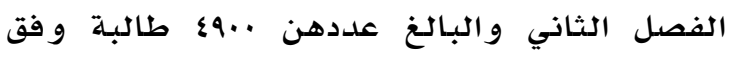
الإحصائيات الصادرة عن دائرة القبول والتئ والتسجيل.

عينة الدراسة

تكونت عينة الدراسـة من • جس طالبـة تم اختيارهن

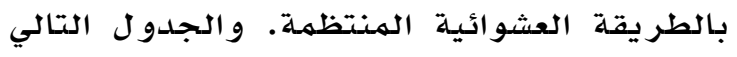
يوضح توزيع أفراد العينة مسب متغيرات

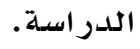

\section{جدول 1}

التكرارات والنسب المئوية حسب متغيرات الدراسة

\begin{tabular}{|c|c|c|c|}
\hline النسبة & التكرار & الفئات & \\
\hline$\wedge ৭, \varepsilon$ & TrY & حائل & \\
\hline $7,1$. & rᄉ & حائل القرى التابعة لمدينة & مكان السكن \\
\hline $\begin{array}{l}1,91 \\
9,1\end{array}$ & $\begin{array}{l}\text { TrA } \\
\text { Tht }\end{array}$ & عززوجة & الحالة الاجتماعية \\
\hline, $1 \ldots$ & r. & المجموع & \\
\hline
\end{tabular}

مقياس التسويف الأكاديهي: عملت الباحثة على تطوير مقياس التسويف الأكاديهي بعد الاطلاع

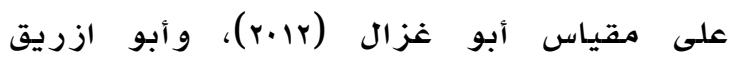

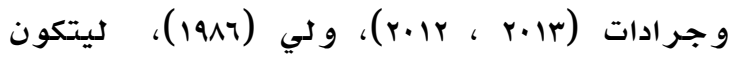

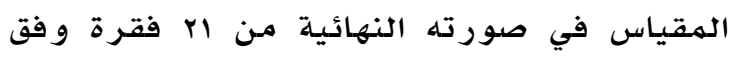

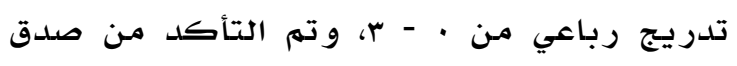

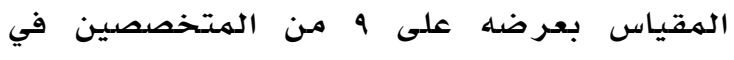

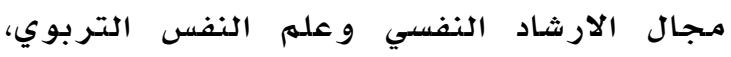
و القياس والتقويم في جامعة حائل، حيث طلب ولبم 
وقد كشفت النتائج عن أن التسويف الأكاديمي

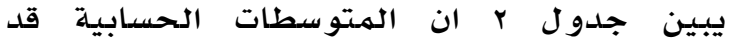

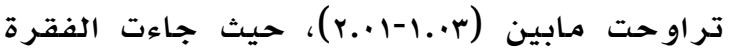

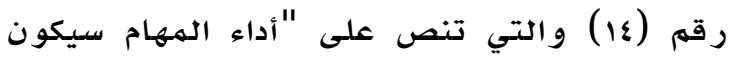

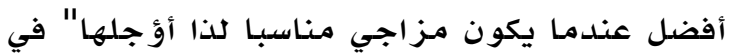

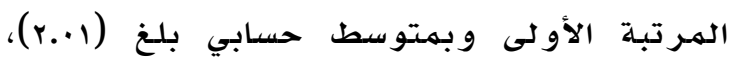

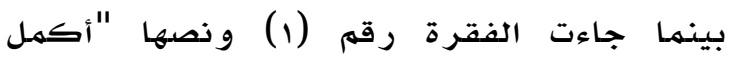
واجباتي بشكل منتظم يوما بيوم، لذا فإنني لا أتأخر في المواد الدراسية" بالمرتبة الأخيرة

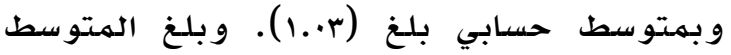
الحسابي للدرجة الكلية (1.0).

مكان السكن (مدينة حائل، القرى التابعة لها)؛ و الحالة الاجتهماعية (متزو جـة، عزباء). المتغير التابع

التسويف الأكاديمي و لله ثلاثة مستو يات: تسويف متدني، و تسو يف متوسط، و تسو يف مـر تفـع. نتائج اللدراسة السؤال الاول: ما نسبـة انتشار التسبويف الأكاديمي لدى طالبات جامعة حائل أ لون لإِجابة عن هذا السؤال تم استخراج المتوسطات الحسابية والانحرافات المعيارية لنسبة انتشار

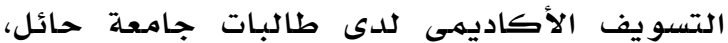

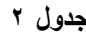

المتوسطات الحسابية والانحرافات المعيارية لفقرات نسبة انتشار التسويف الأكاديمي لاى طالبات جامعة حائل مرتبة تنازليا حسب المتوسطات الحسابية

\begin{tabular}{|c|c|c|c|c|c|}
\hline 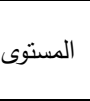 & 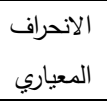 & الحستوسط & 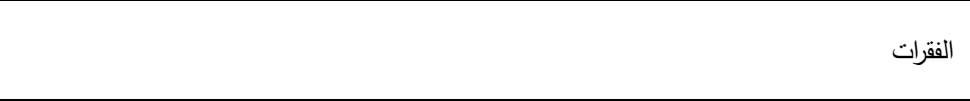 & الرقم & الرتبة \\
\hline متوسط & 9.9 & $\cdot 1, r$ & أداء المهام سيكون أفضل عندما يكون مزاجي مناسبا لذا أؤجلها. & $1 \varepsilon$ & 1 \\
\hline 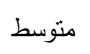 & qVr, & $\cdots, r$ & 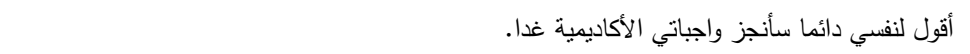 & $\varepsilon$ & r \\
\hline متوسط & $90 \wedge$ & ᄉт, & عندما أواجه مهمات دراسية صعبة أؤمن بضرورة تأجيالها، لأتمكن من معرفة كيفية إنجازها. & ir & r \\
\hline متوسط & $. r+1$ & $\Delta r, 1$ & يعد تأجيل المهمات الأكاديمية مشكلة حقيقية أعاني منها بشكل مستمر . & r. & $\varepsilon$ \\
\hline متوسط & $\cdot r \cdot, 1$ & $\mathrm{vA}, 1$ & إذا انتظرت حتى اللحظة الأخيرة لأنجز مهماتي الدراسية فإنني سأنجزها وكل شيء سيسير على ما يرام. & 19 & 。 \\
\hline متوسط & $\cdots \varepsilon, 1$ & $\checkmark v, 1$ & أنشعر بعدم الراحة من مجرد التفكير بضرورة البدء بإنجاز واجباتي الدراسية. & 17 & ч \\
\hline متوسط & $q \leqslant \vee$ & 10,1 & أقول لنفسي بأنني سأقوم بإنجاز مهماتي الدراسية ثم أتراجع عن ذلك. & 11 & $\checkmark$ \\
\hline متوسط & $1 \times 9,1$ & $.9,1$ & أنا أكون منتجة أكثر عندما أعمل تحت ضغط الوقت ( عندما يقترب وقت الامتحان). & iv & $\wedge$ \\
\hline متوسط & $\cdot \lambda r, 1$ & $0 \wedge, 1$ & أنوقف عن الدراسة في وقت مبكر ؛ لأنها غير ممتعه لكي أقوم بأشياء أكثر متعة. & r) & 9 \\
\hline متوسط & q1r, & $0 \leqslant, 1$ & طويلة. عنتماب موعد الامتحان أجد نفسي منشغلة بأمور أخرى ،فقد ملتت من أداء الواجبات الدراسية لمدة & r & 1. \\
\hline متوسط & $. r v, 1$ & $\varepsilon \wedge, 1$ & أقوم بالعديد من النشاطات الترفيهية بحيث لم ييق لدي الوقت الكافي للاراسة. & 11 & 11 \\
\hline 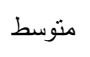 & $. \wedge 1,1$ & $\leq 7,1$ & أؤجل إنجاز المهمات الدراسية بغض النظر عن كونها ممتعة أو غير ممتعة. & 10 & ir \\
\hline متوسط & $91 \cdot$, & r4, & كثيرا ما تمنعني علاقاتي الاجتماعية من القيام بمهامي الدراسية في وقتها. & v & ז \\
\hline منخفض & A.०, & $\cdot r, 1$ & أنهي دائما واجباتي الدراسية المهمة ولدي وقت إضافي " احتياطي". & $* 1$. & $1 \leqslant$ \\
\hline 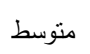 & arr, & rr, & أستعجل عادة لإنجاز المهمات الأكاديمية قبل موعدها المحدد. & *r & 10 \\
\hline متوسط & Nir, & $r \varepsilon, 1$ & أبدأ عادة إنجاز المهمات الدراسية فورا بعد تحديدها. . & *0 & 17 \\
\hline متوسط & $\cdot \leq १, 1$ & r), & ليس مهما إنجاز الواجبات الدراسية في وقتها، فالعالم لن ينتهي إذا لم أنجزها في وقتها. & 9 & iv \\
\hline 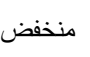 & $.1 \wedge, 1$ & 1०, & أحاول أن أجد لنفسي أعذارا تبرر عدم قيامي بأداء الواجبات الدراسية المطلوبة مني. & $\wedge$ & 11 \\
\hline 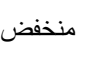 & $\wedge \wedge 9$ & $r, .1$ & أنهي واجباتي الدراسية قبل الوقت المحدد لإنجازها. . & $* 9$ & 19 \\
\hline متوسط & ^९., & 11,1 & ألثزم بالخطة التي أضعها لإنجاز واجباتي الدراسية. & ir & r. \\
\hline \multirow[t]{2}{*}{ 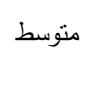 } & Vrr, & $\cdot r, 1$ & أكمل واجباني بشكل منتظم يوما بيوم، لذا فإنني لا أتأخر في المواد الدراسية. & $* 1$ & r) \\
\hline & $\leq \leqslant \vee$ & $0 ., 1$ & 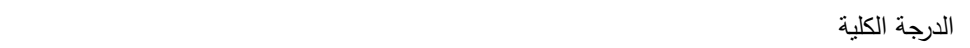 & & \\
\hline
\end{tabular}




\section{مناقشة النتائج}

هدفت الدراسـة الحالية الى التعرف على نسبة انتشار التسويف الأكاديمي للدى طالبات كلية

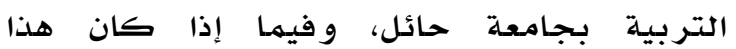
الانتشار يختلف باختلاف مكان السكنة (مدينة

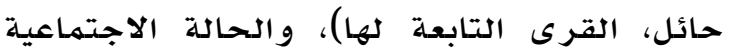

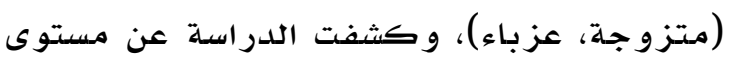

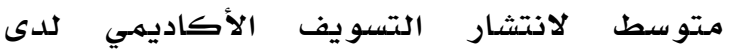
الطالبـات، وبذلك اتفقت الدراسة مـع نظيراتها مـن الدراسات السابقة والتي تبين منها انتشار

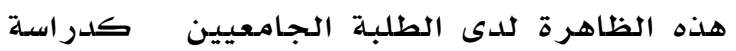

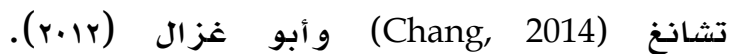
و بذلك تظهر الحاجة لاهتمام المؤسسات

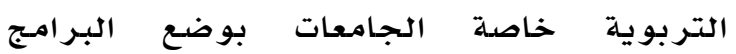
والخطط التي من شأنها خفض مستوى انتشار هذه الظاهرة، لما لها من آثار سلبية على الطالب البـ

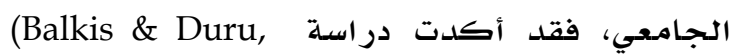
2009, Beck, Koons, \& Migram, 2000)

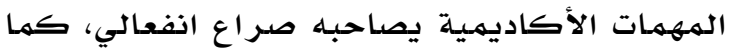
ينتج عنه هبوط في الإنتاجية، وشعور بعدام

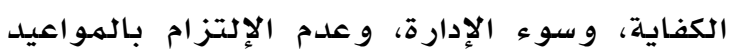
و بالتالي الشعور بالإحباط و الذنب، كما كالإدها كشفت

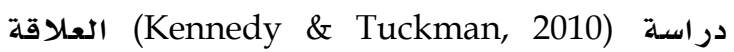
العكسية السلبية بين التسويف الأكاديمي

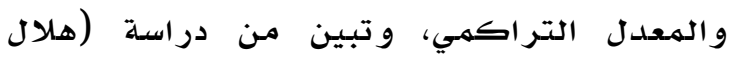

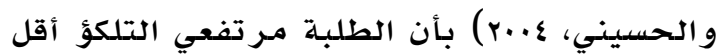

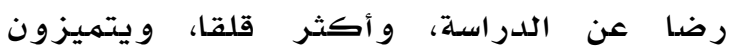

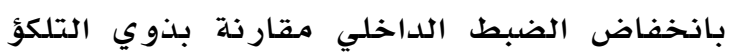
الأكاديهي المنخفض، وأخيرا كشف العنزي و الدغيه (r.r.r) عن علاقة سلبية دالة إحصائيا بين التسويف الدراسي من جهة، و الثقة بالنفس إسله و المعدل الدر اسي من جهة أخرى.
السؤال الثاني: هل تختلف نسبة انتشار التسويف الاكاديهي باختلاف مكان السكن والحالة الاجتماعية؟

جدول ra

المتوسطات الحسابية والانحرافات المعيارية لنسبة انتثار التسويف الاكاديمي حسب متغيرات مكان السكن والحالة الاجتماعية

\begin{tabular}{|c|c|c|c|c|}
\hline العدد & الانحراف المعياري & الحسابي & & \\
\hline MTY & $\cdot, \varepsilon \leqslant V$ & or, 1 & حائل & مكان السكن \\
\hline ऍ & • & $r 1,1$ & لمدينة حائل القرى التابعة & \\
\hline TYA & •, $\leqslant Y \nearrow$ & 01,1 & عزباء & الحالة الاجتماعية \\
\hline Tr & - & $r v, 1$ & متزوجة & \\
\hline
\end{tabular}

للإجابة عن هذا السؤال تم استخراج المتوسطات

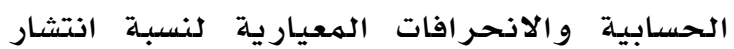
التسويف الاكاديهي حسب متغيرات مكان السكن والحالة الاجتماعية، و جدول r يبين ذلك. يبين جدول r تباينا ظاهريا في المتوسطات الحسابية و والانحر افات المعيارية لنسبة انتشار التسويف الاكاديمي باختلاف فئات متغيرات مكان السكن والحالة الاجتماعية، ولبيان دلادلة الفروق الإحصدائية بين المتوسطات الحسابية تم

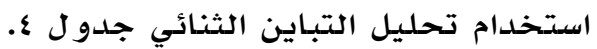
يتبين من جدول ع الآتي:

1. وجود فروق ذات دلالة إحصائية عند

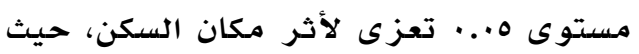

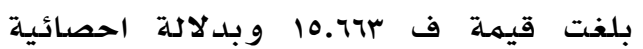
بلغت ...... وجاءت الفروق لصالح حائل. r. عدم وجود فروق ذات دلالة إحصائية عند

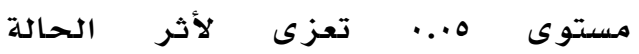

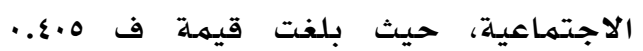

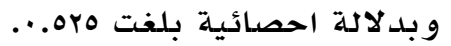

جدول ؛

تحليل التباين الثنائي لأثر مكان السكن والحالة الاجتماعية على نسبة انتثار التسويف الاكاديمي

\begin{tabular}{|c|c|c|c|c|c|}
\hline الدلالة الإحصائية & قيمة ف & متوسط المربعات & درجات الحرية & مجموع المربعات & مصدر التباين \\
\hline$\cdot, \cdots$ & ס & $q \wedge \vee, r$ & 1 & $q \wedge \vee, r$ & مكان السكن \\
\hline \multirow[t]{3}{*}{$\cdot, 0$ Yo } & $\cdot, \varepsilon \cdot 0$ & $\cdot, \cdot V V$ & 1 & $\cdot, \cdot V V$ & الحالة الاجتماعية \\
\hline & & 191, & rov & $\cdot \vee \wedge, \uparrow \wedge$ & الخطأ \\
\hline & & & roq & $.70 \mathrm{~V}, \mathrm{VI}$ & الكلى. \\
\hline
\end{tabular}


مـن خطورتها وتفشيها بين الطلاب، والاهتمام

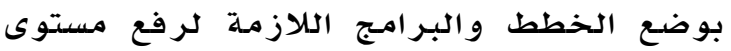
الدافعية لدى الطالبات.

\section{المر اجح}

\section{References}

أبو ازريق، محمدد وجرادات، عبد الكريهم (rابr).

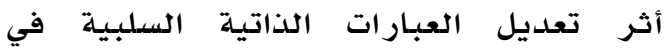
تخفيض التسويف الأكاديمي وتحسين الفاعلية الذاتية الأكاديمية. المجلة الأردية الادنية في العلوم التربوية، و(1)،rV-10. أبو غزال، معاوية (Y.M. r. ). التسويف الأكاديهي:

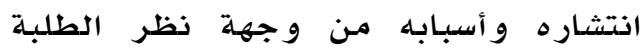

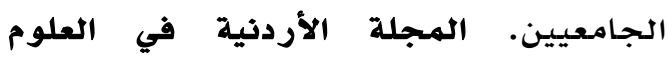

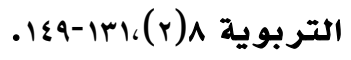

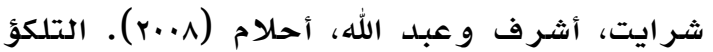

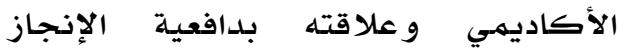

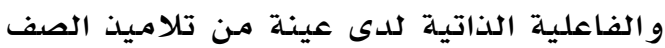
السـادس. مجلة علم النفس المعاصر والعلوم

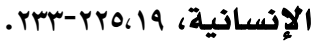

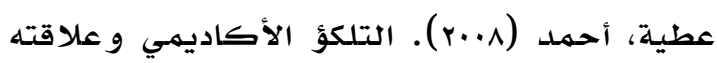

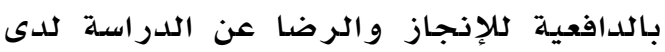
طلاب جامعة الملك خالد بالمـملكة العربية السعودية. المجلة الالكترونية أطفال الخليج ذوي الاحتياجات الخاصة. تاريخ الاسترجاع اليناير 10 بـ، من: العنزي، فريج و الدغيم، محمدل (r..r). سلوك التسدويف الدراسي و علاقتهل ببعض مت بتغير ات الشخصية لدى طلاب كلية التربية الأسـاسية

بالكويت. مجلة كلية التربية- امعة

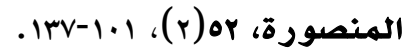

هلال، عبد الرحهن، والحسيني، نادية (ع....).

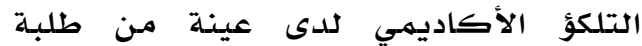

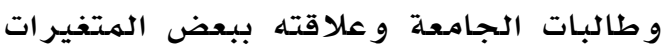

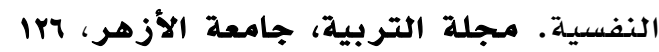
$.1 \varepsilon r-00$ (1)

Balkis, M., \& Duru, E. (2009). Prevalence of academic procrastination behavior among pre-service teachers, and its relationships with demographics and individual
و كشفت الدراسـة اختلاف نسبة انتشار الظاهرة باختالاف مكان السكن حيث كان التسويف مر تفعا للدى ساكنات مدينة حائل عنه لدى ساكنات القرى التابعة لها، وربهما يعلل ذلك بأن ساكنات القرى يشعرن بالضغط لإتمام المهمة الدراسية نظرا لضيق الوقت لديهن؛ حيث يقضين مدة لدئ طويلة تتراوح بين ب-ه ساعات يوميا في السفر من و إلى الجامعة بحسب بعد مكان سكناهن عن مدينة حائل، فتضطر لاتمام مهامها، فيما لهامها يلعب

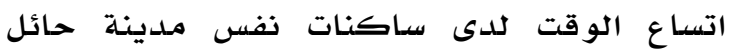
دوره في شعورهن باتساع وقت الهـهمة وبالتالي

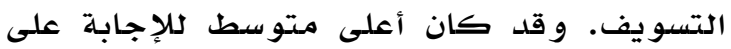
فقر ات المقياس لفقرة (أداء المهام سيكون أفضل ولفول

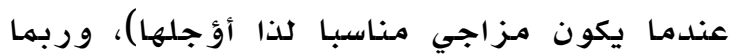
يعود ذلك لحياة الر فاهية التي تعيشها الطالبات

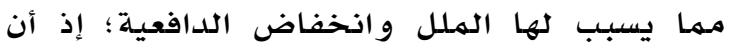
الفرد في كثير من الأحيان يجتهد للحصول

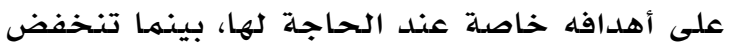
الدافعية لديه عند تحقق كامل رغباته، وبذلك

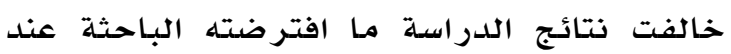

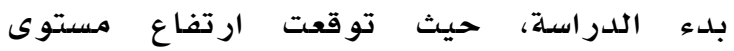

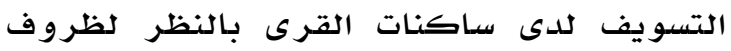

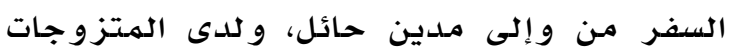
مقارنة بالعزباوات، وربما تعلل هذانه النتيجة

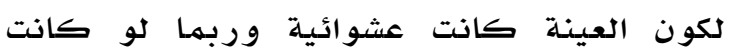
قصدية بحيث تم اختيار العينة من المتزوجات

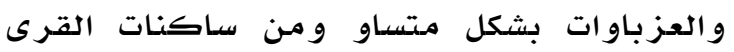

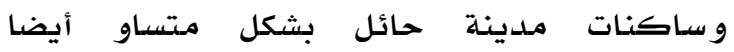
لاختلفت نتائج الدراسة، كما يعلل بأن الباحث

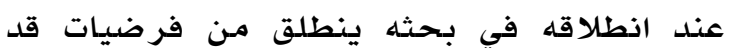
يؤكدها بحثه أو يخالفها.

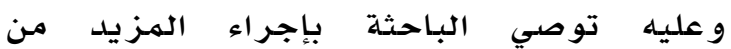
الدراسات حول الظاهرة والأسباب المؤدية إليها، ووضـع البر امـج والخطط العلاجية التي من شأنها

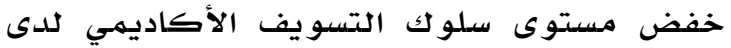
الطالبات، ورفع مستوى الدافعية للإنجاز لديهن. و عليه توصي الباحثة بضرورة اهتمهام الهؤسسات

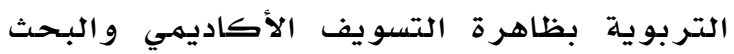

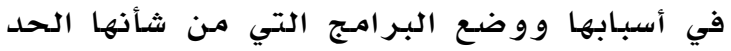


preferences. Journal of Theoyr and Practice in Education, 5(1),18-32.

Balkıs, M,. \& Duru, E. (2010). Akademik erteleme eğilimi, akademik başarı Üniversitesi Eğitim akültesi Dergisi, 27, 159-170.

Beck, B., Koons, S., \& Migram, D. (2000). Correlates and consequences of behavioral procrastination: The effects of academic procrastination, self - consciousness, selfsteam, and self-handicapping. Journal of Social Behavior and Personality, 5, 3-13.

Beswick, G., Rothblum, E.D., \& Mann, L. (1988). Psychological antecedents of student procrastination. Australian Psychologist, 23, 207-217.

Binder, K. (2000). The effects of an academic procrastination treatment on student procrastination and subjective well-being. Unpublished master's thesis, Carleton University. Ottawa, Ontario.

Duru, E., \& Balkis, M. (2014). The roles of academic procrastination tendency on the relationships among self doubt, self esteem and academic achievement. Education and Science, 39, 274-287.

Ellis, A., \& Knaus, W. J. (1977). Overcoming procrastination. New York: Signet Books.

Gard, C. ( 1999). Getting over the "I'll do it tomorrow" blues. Current Health, 2 (26), 22-23.

Gary, J. \& Bruce, W. (2010). The mediating role of procrastination and perceived school belongingness on academic performance in first term freshmen. Paper Given at AERA Annual Meeting, Denver, CO, 2010.

Jaradat, A. (2004). Test anxiety in Jordanian students: Measurement, correlates and treatment. Doctoral dissertation, Phillipsuniversity of Marburg, Germany.

Lay, C.H. (1986). At last, my research article on procrastination. Journal of Research in Personality, 20, 474-495.

Lay, C.H., \& Schouwenburg, H. C. (1993). Trait procrastination, time management, and academic behavior. Journal of Social Behavior Personality, 8, 647-662.

McCown, W., Petzel, T., \& Rupert, P. (1987). An experimental study of some hypothesized behaviors and personality variables of college student procrastinators. Personality and Individual Differences, 8, 781-786.

Milgram, N., Batori, G., Mowrer, D. (1993). Correlates of academic procrastination. Journal of School Psychology, 31(4), 487-500.

Potts, T. J. (1987). Predicting procrastination on academic tasks with self-report personality measures. Doctoral dissertation, Hofstra University.

Piccarelli, R. (2003). How to overcome procrastination The American Salesman, 48 , 27-29.

Rothblum, E. D., Solomon, L. J., \& Murakami, J. (1986). Affective, cognitive, and behavioral differences between high and low procrastinators. Journal of Counseling Psychology, 33, 387-394.

Schouwenburg, H.C. (1992). Procrastinators and fear of failure: An exploration of reasons for procrastination. European Journal of Personality, 6, 225-236.

Semb, G. Glick, D. M., \& Spencer, R. E. (1979). Student withdrawals and delayed work patterns in self-paced psychology courses. Teaching of Psychology, 6, 23-25.

Szalaeitz, M. (2003). Stand and deliver. Psychology Today, 36(4), 50-53.

Tice, D. M., \& Baumeister, R. F. (1997). Longitudinal study of procrastination, performance, stress, and health: The costs and benefits of dawdling. Psychological Science, 8, 454-458.

Jackson, T. (2003). Procrastination and perceptions of past, present, and future Individual differences research group Retrieved on February 11, 2015. From: www.idr-journal.com

Özer, B., Demir, A., \& Ferrari, J. (2009). Exploring academic procrastination among Turkish students: possible gender differences in prevalence and reasons. The Journal of Social Psychology, 149 (2), 241-257.

Wolters, C.A. (2003). Understanding procrastination from self-regulated learning perspective. A Journal of Educational psychology, 95 (1) 179-205. 